\title{
Anaphylaxis After Ingesting Ibuprofen Liquid Gelatin-Capsule
}

\author{
Fahad Al-Ghimlas ${ }^{1, *}$, Nasser Al-Ahmed ${ }^{2}$ and Sean Mace $^{3}$
}

${ }^{I}$ Chest Unit, Department of Medicine, Amiri Hospital, Ministry of Health, State of Kuwait

2Department of Allergy, Al-Rashed Allergy Centre, Ministry of Health, State of Kuwait

${ }^{3}$ Division of Allergy and Clinical Immunology, Department of Medicine, St. Michael's Hospital, University of Toronto, Canada

\begin{abstract}
Non-steroidal anti-inflammatory drugs are used daily by millions of patients worldwide for the management of various inflammatory diseases. Many well-documented adverse reactions are related to the use of these drugs. We report a fifty-four year-old woman with anaphylaxis after ingestion of ibuprofen liquid in a gelatin capsule. Eventually this was concluded to have resulted from hypersensitivity to the gelatin component of the capsule, which was likely IgE-mediated because of the positive skin test to gelatin. Gelatin allergy is only relevant for patients ingesting specific capsule formulation. The allergist/clinical immunologist must keep in mind the possibility of gelatin allergy.
\end{abstract}

Keywords: Anaphylaxis, Gelatin, Ibuprofen, Non-steroidal anti-inflammatory drugs.

\section{INTRODUCTION}

Non-steroidal anti-inflammatory drugs (NSAIDs) play an enormous role in the management of inflammatory diseases and as analgesics. NSAIDs are used daily by millions of patients globally. Knowledge of the complete spectrum of potential adverse reactions to these medications is important for the practicing allergist/ clinical immunologist $[1,2]$. Newer formulations available further complicate the evaluation of adverse reactions that occur as a result of their use. We present a case of anaphylaxis after ingestion of ibuprofen in a gelatin capsule.

\section{CASE REPORT}

A fifty-four years old woman referred to the clinic for further assessment of possible allergy to tomatoes. She reported rhinoconjunctivitis, chest tightness, and angioedema within minutes from ingesting freshly prepared tomatoes. These symptoms settled with an antihistamine. The patient had no previously known food or medication allergies. She recalled having ingested cucumber and ibuprofen around the same time as having fresh tomato. Her past medical history is significant for asthma controlled with inhaled steroids and occasional short acting beta-agonists. She infrequently requires analgesics for occasional headaches. Family, social and environmental histories were non-contributory. Physical examination at the time of consultation revealed within normal vital signs. The head and neck, as well as skin examinations were normal. Cardiovascular examination was unremarkable and chest auscultatory notes were normal. Allergy skin testing was done to commercial, as well as freshly pre pared tomato and cucumber products. Tests were all negative

*Address correspondence to this author at the Chest Unit, Department of Medicine, Amiri Hospital, P.O.Box 39810 Nuzha, Postal Code 73059; Tel: +(965) 22464725; Fax: +(965) 22435861;

E-mail: dr_alghimlas@yahoo.com with a positive histamine control. The patient was challenged with oral ingestion of tomatoes and had developed no symptoms after a total observation period of 4 hours. Furthermore, to rule out unidentified food additives, the patient was challenged with ingestion of specific cultivars of tomatoes and cucumber that were suspected of causing the reaction. This testing was also negative.

At this point, food allergy was deemed unlikely and consideration was given to possible direct NSAID sensitivity, namely ibuprofen. The patient was asked to bring the specific brand of ibuprofen she had been using to the clinic on the next visit. On her follow-up visit, she reported development of a similar but more severe reaction, associated with hypotension; i.e: grade 3 anaphylaxis [3], after ingesting only one tablet of the same drug with no other co-ingestions. This episode required epinephrine administered by attending paramedics. The ibuprofen was found to be a "gel-cap" preparation (Pfizer, USA). The capsule was made of bovine gelatin.

Skin testing by prick method done with the drug liquid aspirated from the capsule was negative. Skin testing with a dissolved whole ibuprofen gel-cap was positive. She had a positive skin test when tested with commercial bovine gelatin only (Fig. 1). Oral challenge with regular ibuprofen, without gelatin capsule and this was negative. Given the clearly positive skin prick tests to the gelatin an incremental oral challenge was not considered at that point. Since gelatin is also available as a food ingredient, we later called the patient for re-evaluation. However, due to failure to follow-up, we were unable to evaluate for reactions to other gelatincontaining food or medical products, neither we were able to evaluate for in vitro specific-IgE against bovine gelatin.

\section{DISCUSSION}

NSAIDs are associated with adverse reactions involving cutaneous, respiratory, cardiovascular, and gastrointestinal 


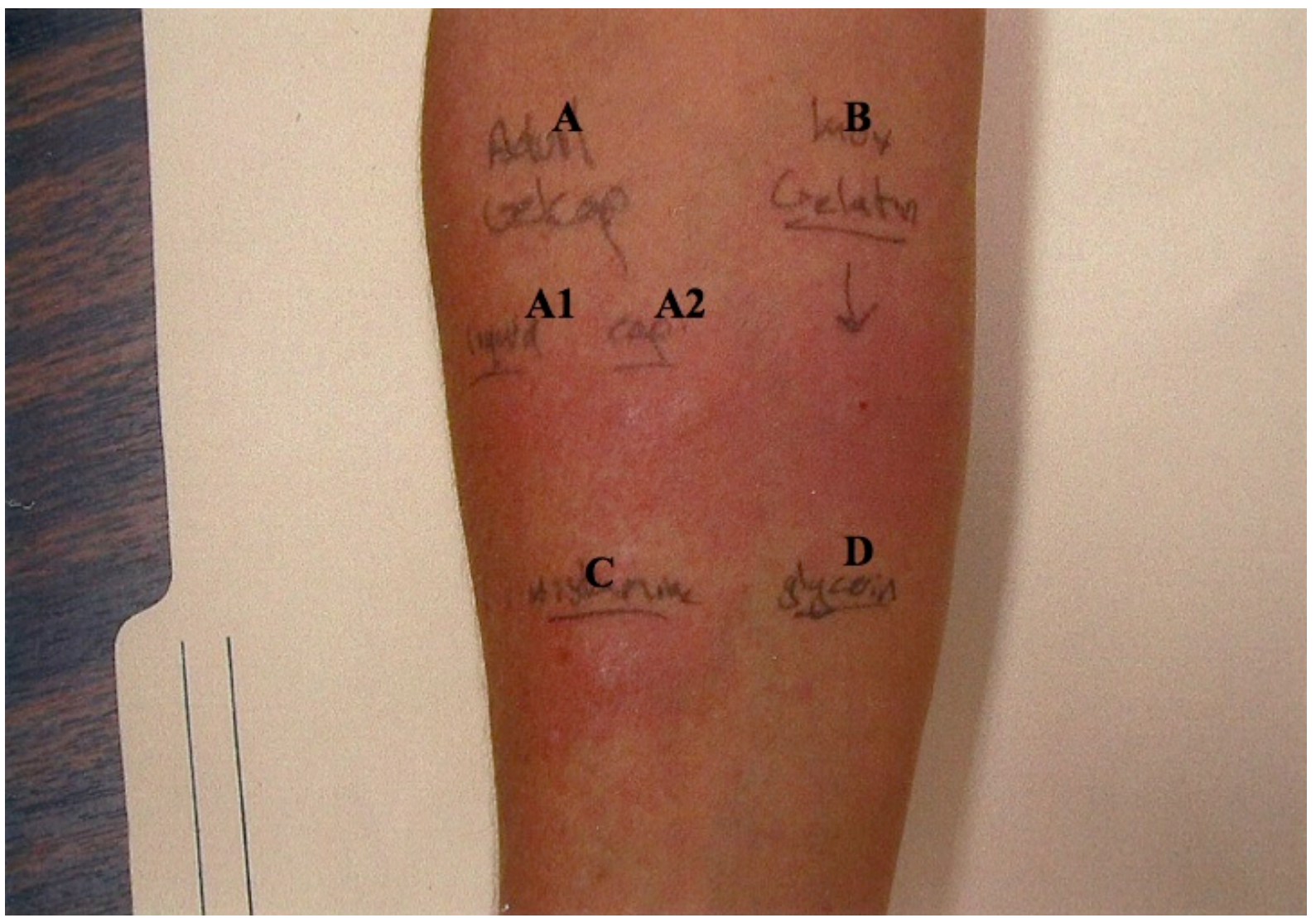

Fig. (1). Positive skin prick test to both gelatin and dissolved ibuprofen including the gel-capsule from Advil Liqui-Gel® product.

Wheal size (in millimeters):

A: Advil gel-capsule: A1: Liquid, $1 \mathrm{~mm}$; A2: Cap (gel-capsule), $7 \mathrm{~mm}$; B: Gelatin, $6 \mathrm{~mm}$; C: Histamine (positive control), 5 mm; D: Glycerin (negative control), $1 \mathrm{~mm}$.

systems. These reactions range from urticaria and angioedema to anaphylaxis [1,2]. Available evidence is limited with respect to the relative safety of these drugs. There are different families of NSAIDs and within these families there are differences between individual agents with diclofenac, naproxen, and ibuprofen having increased risk of allergic adverse reactions, diclofenac having the highest [4].

On the other hand, allergic reactions to gelatin had been described thoroughly in the medical literature. Gelatin is primarily used in vaccines as stabilizers. IgE-mediated systemic reactions to gelatin resulting in anaphylactic reactions to Measles, Mumps and Rubella (MMR) vaccine [5] and urticaria following varicella vaccine [6] have been described. In both cases $\operatorname{IgE}$ antibodies to gelatin were recorded [5, 7]. There are also reports of IgE- and non-IgE-mediated allergic reactions attributed to gelatin in foods (candy fruit chews and gummy bears) $[8,9]$ and in other medical products including intravenous fluids (modified fluid gelatins) [10], chloral hydrate suppositories [11], erythropoietin [12], and a gelatin-containing surgical sponge [13].

This patient likely had an IgE-mediated reaction to gelatin as documented by a positive skin test to this product. This was proven by a positive test to the whole capsule, but not to the carefully aspirated liquid medication, namely ibuprofen, and with a negative challenge to the same gel-free drug. Positive result after gelatin skin testing using Jell-O sugared gelatin (Kraft Foods, Rye Brook, N.Y., USA), as previously described [14], confirms an underlying IgE-mediated hypersensitivity to this ingredient.

Due to failure to follow-up, we were unable to evaluate for reactions to other gelatin containing food or medical products, neither we were able to evaluate for in vitro specific-IgE against bovine gelatin. Also, we were unable to differentiate reactions peculiar to such form of gelatin; i.e. bovine versus porcine. The newer ibuprofen product, called Advil Liqui-Gel ${ }^{\circledR}$, is composed of an outer casing of Gelatin filled with solubilized ibuprofen. The manufacturer claims that such preparation offer the consumer a fast pain relief compared to the older products available in the market.

\section{CONCLUSION}

With the increased use of these newer preparations of analgesics, i.e: ibuprofen with gelatin capsule, the allergist/ clinical immunologist must keep in mind the possibility of a gelatin-induced allergic reaction when evaluating patients presenting with an adverse reaction following the ingestion of such products. Moreover, as bovine gelatin is present in different manufactured items, including foods, drugs, and vaccines, it is important to evaluate for reactions to these products.

\section{CONFLICT OF INTEREST}

The authors confirm that this article content has no conflicts of interest. 


\section{ACKNOWLEDGEMENT}

Declared none.

\section{REFERENCES}

[1] van der Klauw MM, Stricker BH, Herings RM, Cost WS, Valkenburg HA, Wilson JH. A population based case-cohort study of drug-induced anaphylaxis. Br J Clin Pharmacol 1993; 35:400-8. der Klauw MM, Wilson JH, Stricker BH. Drug-associated anaphylaxis: 20 years of reporting in the Netherlands (1974-1994) and review of the literature. Clin Exp Allergy 1996; 26:1355-63.

[3] Simon G.A. Brown. Clinical features and severity of anaphylaxis. J Allergy Clin Immunology 2004;114:371-6.

[4] van Puijenbroek EP, Egberts AC, Meyboom RH, Leufkens HG. Different Risks for NSAID-Induced Anaphylaxis. Ann Pharmacother 2002; 36:24-9.

[5] Kelso JM, Jones RT, Yunginger JW. Anaphylaxis to measles, mumps, and rubella vaccine mediated by IgE to gelatin. J Allergy Clin Immunol 1993; 91: 867-72.

[6] Singer S, Johnson CE, Mohr R, Holowecky C. Urticaria following varicella vaccine associated with gelatin allergy. Vaccine 1999; 17: 327-9.
[7] Sakaguchi M, Yamanaka T, Ikeda K, et al. IgE-mediated systemic reactions to gelatin included in the varicella vaccine. J Allergy Clin Immunol 1997; 99: 263-4.

[8] Wang J, Sicherer SH. Anaphylaxis following ingestion of candy fruit chews. Ann Allergy Asthma Immunol 2005; 94: 530-3.

[9] Scurlock AM, Althage KA, Christie L, Burks AW, Jones SM. Anaphylaxis after ingestion of gummy bears. J Allergy Clin Immunol 2002; 110: 936-7.

[10] Vervloet D, Senft M, Dugue P, Arnaud A, Charpin J. Anaphylactic reactions to modified fluid gelatins. J Allergy Clin Immunol 1983; 71: 535-40.

[11] Yamada A, Ohshima Y, Tsukahara H, et al. Two cases of anaphylactic reaction to gelatin induced by a chloral hydrate suppository Pediatr Int 2002; 44: 87-9.

[12] Sakaguchi M, Kaneda H, Inouye S. A case of anaphylaxis to gelatin included in erythropoietin products. J Allergy Clin Immunol 1999; 103: 349-50.

[13] Puerello-D Ambrosio F, Gangemi S, La Rosa G, Merendino RA, Tomasello F. Allergy to gelatin. Allergy 2000; 55: 414-5.

[14] Coop CA, Balanon SK, White KM, Whisman BA, Rathkopf MM. Anaphylaxis from the Influenza Virus Vaccine. Int Arch Allergy Immunol 2008; 146: 85-8.

Received: November 13, 2012

Revised: December 02, 2012

Accepted: December 08, 2012

(C) Al-Ghimlas et al.; Licensee Bentham Open.

This is an open access article licensed under the terms of the Creative Commons Attribution Non-Commercial License (http://creativecommons.org/licenses/by-nc/3.0/) which permits unrestricted, non-commercial use, distribution and reproduction in any medium, provided the work is properly cited. 